\title{
JOIN-IRREDUCIBLE CROSS PRODUCT VARIETIES OF GROUPS $\left({ }^{1}\right)$
}

\author{
BY
}

JAMES J. WOEPPEL

\begin{abstract}
Let $\mathfrak{U}, \mathfrak{B}$ be varieties of groups which have finite coprime exponents, let $u$ be metabelian and nilpotent with "small" nilpotency class, and let $\mathfrak{B}$ be abelian. The product variety $\mathcal{U}_{\mathfrak{B}}$ is shown to be join-irreducible if and only if $\mathfrak{U}$ is join-irreducible. This is done by obtaining a simple description for the critical groups generating $\mathfrak{u} B$ when $\mathfrak{u}$ is join-irreducible and finding a word which is not a law in $\mathfrak{U B}_{\mathfrak{B}}$ but is a law in every proper subvariety of $\mathfrak{U B}$.
\end{abstract}

1. Introduction. (Throughout this paper any terminology or notation which is unexplained in the paper is used as described in Hanna Neumann's book [5].) Because every Cross variety contains only finitely many isomorphism classes of critical groups (see $[5,51.51]$ ), every Cross variety can be written as a (finite) join of join-irreducible subvarieties. The work of Brisley [1] and Weichsel [8] gives an explicit join decomposition into join-irreducible varieties for varieties of finite exponent which are metabelian and nilpotent with nilpotency class less than any prime divisor of the exponent (i.e. metabelian of small class). It follows from their results that the join-irreducible varieties of this kind are of the form $\mathscr{A} \mathscr{A} \wedge \mathfrak{N}_{c} \wedge \mathfrak{B}_{q}$ where $q$ is a prime power. In this paper we obtain a similar result for Cross product varieties whose first factor is of the type treated by Brisley and Weichsel. Šmel'kin (see [5, 24.64]) has shown that a Cross variety is a proper product variety if and only if it is a product of two nontrivial varieties with relatively prime exponents, the first variety nilpotent and the second variety abelian.

For positive integers $n, c$ with $c$ less than every prime divisor of $n$ let $\mathfrak{N}_{c, n}=\mathscr{U} \mathfrak{A} \wedge \mathfrak{N}_{c} \wedge \mathfrak{B}_{n}$. A variety is metabelian of small class if it is a subvariety of some $\mathfrak{M l}_{c, n}$.

Presented to the Society, August 6, 1970 under the title Joint-irreducible cross product varieties; received by the editors October 20, 1972.

AMS (MOS) subject classifications (1970). Primary 20E10; Secondary 20E25.

Key words and phrases. Varieties of groups, join-irreducible varieties, product varieties, Cross varieties, critical group, nilpotent by abelian.

(1) The results contained in this paper are from the author's Ph.D. dissertation [9], submitted to the University of Illinois at Urbana-Champaign, 1970. 
THEOREM I. Let $\mathfrak{U}$ be a nontrivial metabelian variety of small class and let $m$ be a positive integer relatively prime to the exponent of $\mathfrak{U}$. The variety $\mathfrak{U A}_{m}$ is join-irreducible if and only if $\mathfrak{U}$ is join-irreducible; that is $\mathfrak{U}=\mathfrak{M} \mathfrak{R}_{c, q}$ for some prime power $q$.

The case $c=1$ has been proved by Cossey [4, p. 43], and the case $c=$ 2, $\mathfrak{U}$ of prime exponent $p$ and $m$ a prime divisor of $p-1$ has been proved by Burns [3, Lemma 6.7(3)]. Hence throughout the rest of this paper $c$ will denote a positive integer greater than 1 .

The only if part of the theorem follows immediately from 21.23 of [5] and the results of Brisley and Weichsel. The if part is proved by analyzing the structure of the critical groups generating $\mathfrak{M} l_{c, q} \mathfrak{A}_{m}$-here $q$ is a power of an odd prime $p$, the positive integers $m, p$ are relatively prime and $c$ is less than $p$.

The results of this analysis may be summarized as follows:

THEOREM II.(2) (i) Let $C$ be a critical group in $\mathfrak{N}_{c, q} \mathfrak{A}_{m}$ which is not in $\mathfrak{A}_{m}$. The Fitting radical, Fit $C$, of $C$ lies in $\mathfrak{M}_{c, q}$ and $C /$ Fit $C$ is a subgroup of a c-fold direct power of a cyclic group of order $m$.

(ii) There is a proper subvariety of $\mathfrak{M}_{c, q} \mathfrak{A}_{m}$, which contains all the critical groups $C$ of $\mathfrak{N R}_{c, q} \mathfrak{Q}_{m}$ in which either (a) the exponent of the cth term of the lower central series of Fit $C$ is less than $q$ (that is, Fit $C$ does not generate $\mathfrak{M l}_{c, q}$ ), or (b) the order of $C /$ Fit $C$ is less than $m^{c}$.

(iii) A critical group $C$ generates $\mathfrak{M l}_{c, q} \mathfrak{H}_{m}$ if Fit $C$ generates $\mathfrak{M}_{c, q}$ and $C /$ Fit $C$ is a $c$-fold direct power of a cyclic group of order $m$.

2. Proof of Theorem II. For notational convenience we put $\mathfrak{U}=\mathfrak{M}\}_{c, q}$ and $\mathfrak{B}=\mathfrak{U A}_{m}$.

Let $C$ be a critical group in $\mathfrak{B}$. The discussion in the proof of 2.1 of Burns' paper [2] yields the following results: The Fitting radical $F$ of $C$ lies in $\mathfrak{U}$. There are $s$ (not exceeding $c$ ) normal subgroups $M_{1}, \cdots, M_{s}$ of $C$ containing the Frattini subgroup $\Phi$ of $C$ such that $F=M_{1} \cdots M_{s}$ and . the $M_{i} / \Phi$ are minimal normal subgroups of $C / \Phi$. There is a complement $L$ for $F$ in $C$. The intersection of the centralizers $C_{i}$ of $M_{i} / \Phi$ in $L$ is trivial and $L / C_{i}$ is cyclic of order $m_{i}$ dividing $m$. Part (i) of Theorem II follows at once from these results.

The key to the proof of part (ii) of Theorem II is the word $\omega$ defined in the next paragraph. We will show that $\omega$ is not a law in $\mathfrak{Z}$. Later we will further show that every critical group in $\mathfrak{B}$ which does not have $\omega$ as a law generates the variety $\mathfrak{W}$ (this is part (iii) of Theorem II).

(2) The statement of Theorem II was suggested by the referee. 
We now define the word $\omega:$ For $i$ in $\{1, \cdots, c\}$ put

$$
\mu_{i 1}=x_{i 1}^{m}, \quad \mu_{i 2}=\left[\mu_{i 1}, x_{i 2}\right]
$$

and for $n$ in $\{3, \cdots, m\}$ put

$$
\mu_{i n}=\left[\mu_{i, n-1}, x_{i 2}^{-1} x_{i n}, \cdots, x_{i, n-1}^{-1} x_{i n}\right]
$$

also put

$$
\mu_{i}=\left[\mu_{i m}, \cdots, x_{i n}^{-1} x_{j l}, \cdots\right]
$$

where $n, l \in\{2, \cdots, m\}, j \in\{1, \cdots, c\} \backslash\{i\}$ and the quotients are, for definiteness, taken in lexicographic order. Finally, put

$$
\omega=\left[\mu_{1}, \cdots, \mu_{c}\right]^{q / p} .
$$

Let $k$ be the smallest positive integer such that $m$ divides $p^{k}-1$ and let $F_{k}$ be a relatively free group of rank $k$ of the variety $\mathfrak{U}$. The Frattini quotient group $F_{k} / \Phi\left(F_{k}\right)$ has an automorphism $\beta^{\prime}$ of order $m$; this fact follows from the results of M. F. Newman in [6]. Because $F_{k}$ is relatively free, there is an endomorphism $\epsilon$ of $F_{k}$ which induces $\beta^{\prime}$ on $F_{k} / \Phi\left(F_{k}\right)$. The endomorphism $\epsilon^{m}$ induces the identity on $F_{k} / \Phi\left(F_{k}\right)$. Since $F_{k}$ is a finite $p$-group, $\epsilon^{m}$ is an automorphism of $F_{k}$ of $p$-power order. Hence there is a power of $\epsilon$ which is an automorphism of order $m$ of $F_{k}$ that induces $\beta^{\prime}$ on $F_{k} / \Phi\left(F_{k}\right)$; denote this automorphism by $\beta .(3)$ There is a generating set $\left\{y_{1}, \cdots, y_{k}\right\}$ of $F_{k}$ such that $y_{1} \beta=y_{2}, \cdots, y_{k-1} \beta=y_{k}$.

Let $F_{c k}$ be a relatively free group of the variety $U$ freely generated by $\left\{y_{11}, \cdots, y_{1 k}, y_{21}, \cdots, y_{c k}\right\}$. There are automorphisms $\beta_{1}, \cdots, \beta_{c}$ of $F_{k}$ each of order $m$ such that $\beta_{i}$ fixes each $y_{j n}$ with $j \neq i$ and acts on $\left\{y_{i 1}, \cdots, y_{i k}\right\}$ as $\beta$ acts on $\left\{y_{1}, \cdots, y_{k}\right\}$. Let $B$ be the abelian subgroup of the automorphism group generated by $\left\{\beta_{1}, \cdots, \beta_{c}\right\}$ and let $V$ be the splitting extension of $F_{c k}$ by $B$. Clearly $V$ lies in $\mathfrak{W}$. Under the substitution

$$
x_{i 1} \mapsto y_{i 1}, \quad x_{i n} \mapsto \beta_{i}^{n-1}, \quad n \geqslant 2,
$$

the value of $\omega$ in $V$ is not the identity; thus $\omega$ is not a law in $\mathfrak{W}$.

Let $C$ be a critical group in $\mathbb{B}$ which does not have $\omega$ as a law. Clearly, the $c$ th term of the lower central series of the Fitting radical $F$ of $C$ has exponent precisely $q$. Since $\omega$ is not a law in $C$, there is a substitution for the $x_{11}, \cdots, x_{c m}$ in $\omega$ with values in $C$ such that $\omega$ is not the identity. Under this substitution $\mu_{1}$ has a value in $C$ outside of $\Phi$. There is no loss of generality in labeling the $M_{i}$ so that the value of $\mu_{1}$ lies outside of

(3) The author is indebted to the referee for suggesting this simplified argument for the existence of $\beta$. 
$M_{2} \cdots M_{s}$. Hence the value of $\mu_{11}$ lies outside of $M_{2} \cdots M_{s}$; each of $x_{12}, \cdots, x_{1 m}$ have corresponding values in distinct nontrivial cosets of $F C_{1}$ in $C$ and the corresponding values of $x_{j n}$ for $j \neq 1$ and $n \neq 1$ lie in $F C_{1}$. Hence $C / F C_{1}$ has order $m$. This argument can be continued to show that $C / F\left(C_{1} \cap \cdots \cap C_{i}\right)$ has order $m^{i}$ and the corresponding values of $x_{j n}$ $(j>i$ and $n \neq 1)$ lie in $F\left(C_{1} \cap \cdots \cap C_{i}\right)$. It follows that $F / C$ has order $m^{c}$ and so the proof of part (ii) of Theorem II is complete.

We now establish part (iii) of Theorem II; assume now that $C$ is a critical group in $\mathfrak{W}$, Fit $C$ generates $\mathfrak{M} l_{c, q}$, and $C /$ Fit $C$ is a $c$-fold direct power of a cyclic group of order $m$. Note that by part (i) of Theorem II and the facts stated in the above paragraph, any critical group in $\mathfrak{B}$ which does not have $\omega$ as a law satisfies these hypotheses.

A generating set $\left\{a_{1}, \cdots, a_{c}\right\}$ for $L$, the complement of the Fitting radical $F$ of $C$, can be chosen so that each $a_{i}$ has order $m$ and

$$
a_{i} \in C_{i} \cap \cdots \cap C_{i-1} \cap C_{i+1} \cap \cdots \cap C_{c} .
$$

Moreover $F / \Phi$ is the direct product of $M_{1} / \Phi, \cdots, M_{c} / \Phi$ and each $M_{i} / \Phi$ has order $p^{k}$ because it admits a faithful representation of the cyclic group $L / C_{i}$ of order $m$. Thus it is possible to select $z_{i}$ in $M_{i}$ so that $\left\{z_{1}, \cdots, z_{c}\right.$, $\left.a_{1}, \cdots, a_{c}\right\}$ generates $C$ and $\left\{z_{11}, \cdots, z_{1 k}, z_{21}, \cdots, z_{c k}\right\}$ generates $F$ wher $z_{i 1}=z_{i}$ and $z_{i n}=a_{i}^{-1} z_{i, n-1} a_{i}$ for $n \in\{2, \cdots, k\}$.

As before let $F_{c k}$ be a relatively free group of $\mathfrak{U}$ generated by $\left\{y_{11}, \cdots, y_{c k}\right\}$. Let $\psi$ be a homomorphism from $F_{c k}$ to $F$ such that $y_{j n} \psi=z_{j n}$. Let $\alpha_{i}^{\prime}$ be an endomorphism of $F_{c k}$ defined by

$$
\left(y_{j n} \alpha_{i}^{\prime}\right) \psi=a_{i}^{-1} z_{j n} a_{i}
$$

Clearly $\left(\alpha_{i}^{\prime}\right)^{m}$ induces the identity automorphism on $F_{c k} / \Phi\left(F_{c k}\right)$ and so, arguing as before, there is an automorphism $\alpha_{i}$ of $F_{c k}$ having order $m$ such that

$$
\left(y_{j n} \alpha_{i}\right) \psi=a_{i}^{-1} z_{j n} a_{i}
$$

Let $A$ be the abelian subgroup of the automorphism group of $F_{c k}$ generated by $\left\{\alpha_{1}, \cdots, \alpha_{c}\right\}$. It follows that $C$ is a quotient group of the splitting extension of $F_{c k}$ by $A$.

It is not too difficult to verify that $A$ and the group $B$ defined earlier are conjugate in the automorphism group of $F_{c k}$; (this argument follows from the fact that the images of $A$ and $B$ in the general linear group $\operatorname{GL}(c k, p)$ are conjugate). Hence the splitting extension of $F_{c k}$ by $A$ is isomorphic to 
$V$ (see Theorem 3.3 in Taunt [7]). Therefore every critical group in 23 which satisfies the hypotheses of part (iii) of Theorem II is isomorphic to a quotient group of $V$.

A similar argument shows that every critical group in $\mathfrak{W}$ is isomorphic to a section (factor) of $V$. This may be compared with Lemma 2.6 in [2].

It thus follows that $V$ generates the variety $\mathfrak{B}$.

The next step is to show that a certain quotient group of the group $V$ still generates $\mathfrak{W}$. For each sequence $\pi=\left(\pi_{1}, \cdots, \pi_{c}\right)$ of nonnegative integers such that $\pi_{1}+\cdots+\pi_{c}=c$, a subgroup $M(\pi)$ of $V$ is defined by: $M(\pi)$ contains all commutators $\left[y_{i_{1} n_{1}}, \cdots, y_{i_{s} n_{s}}\right]$ which have for some $i$ in $\{1, \cdots, c\}$ more than $\pi_{i}$ of $\left\{i_{1}, \cdots, i_{s}\right\}$ equal to $i$, and $M(\pi)$ contains all $\beta_{i}$ for which $\pi_{i}=0$. It is easy to check that the $M(\pi)$ are normal subgroups of $V$. One can also verify by a straightforward argument that their intersection is trivial. Hence the variety $\mathfrak{W}$ is generated by the set of quotient groups $V / M(\pi)$. Unless $\pi=(1, \cdots, 1)$ the Fitting quotient group of $V / M(\pi)$ has order less than $m^{c}$ and so $\omega$ is a law in $V / M(\pi)$. Thus one is lead to investigate the quotient group $V / M$ where $M=M(1, \cdots, 1)$ more closely. It is straightforward to check that for each $\pi$ the mapping from $V$ to $V / M$ determined by: for $\pi_{i}=0$

$$
y_{i 1} \mapsto M, \quad \beta_{i} \mapsto M ;
$$

otherwise (i.e. for $\pi_{i} \neq 0$ )

$$
y_{i 1} \mapsto y_{D(i), 1} \cdots y_{U(i), 1} M, \quad \beta_{i} \mapsto \beta_{D(i)} \cdots \beta_{U(i)}
$$

where

$$
D(i)=\sum_{j=1}^{i-1} \pi_{j}+1 \text { and } U(i)=\sum_{j=1}^{i} \pi_{j}
$$

is a homomorphism with kernel $M(\pi)$. Therefore $V / M$ generates $\mathfrak{W} .\left(^{(4)}\right.$

Also each critical group in $\mathfrak{W}$ which satisfies the hypotheses of part (iii) of Theorem II is isomorphic to a quotient group of $V / M$; thus, in particular, any critical group in which $\omega$ is not a law is isomorphic to a quotient group of $V / M$. For it was shown above that such a critical group $C$ is isomorphic to a quotient group $V / K$ with $K$ a normal subgroup of $V$ contained in $\Phi\left(F_{c k}\right)$. The normal subgroup $K$ contains the subgroup $M$; because if $M$ is not contained in $K$ then one can show that this critical group $C$ is isomorphic to a section (factor) of an external direct product of proper subgroups of $V / K$ which is a contradiction. The factors in this external direct product are the proper sub-

(4) The argument presented in this paragraph was suggested by the referee; it simplifies the author's original argument considerably. 
groups $\left(D_{i} K\right) \circ B / K$ of the group $V / K$ where $D_{i}$ is a subgroup generated by the set,

$$
\left\{y_{j}^{\beta} \mid j \text { appears as a subscript in } d_{i}, \beta \in B\right\},
$$

of elements of $V$ with $\left\{d_{1}, \cdots, d_{l}\right\}$ the set of left normed commutators in $M$ but not in $K$ (assuming $M$ is not contained in $K$ ).

The final step in the proof will be to show that the socle, $S / M$, of $V / M$ is minimal characteristic in $V / M$. For then $V / M$ must lie in the variety generated by any critical group in $\mathfrak{W}$ which satisfies the hypotheses of part (iii) of Theorem II, and part (iii) will follow. It also follows that every critical group which does not have $\omega$ as a law generates the variety 23 .

To show that $S / M$ is minimal characteristic, it is necessary to introduce some automorphisms of $V / M$. Let $\Phi$ be the Frattini subgroup of $V$ and $V_{i}$ the subgroup generated by $\left\{y_{i 1}, \cdots, y_{i k}\right\}$ and $\Phi$. The group $V_{i} / \Phi$ can be thought of as the additive group of a field $K$ of $p^{k}$ elements and the subgroup $B_{i}$ generated by $\beta_{i}$ as a subgroup of the multiplicative group of the field $K$ (cf. [6]). The automorphism $x \mapsto x^{p}$ of this field $K$ can, in an obvious way, be extended to an automorphism of the splitting extension of $V_{i} / \Phi$ by $B_{i}$. It can be further extended to an automorphism $\gamma_{i}$ of $V$ which induces the identity on each of the $V_{j} / \Phi$ and $B_{j}$ for $j \neq i$. Clearly $M \gamma_{i}=M$ so $\gamma_{i}$ induces an automorphism on $V / M$. Also every permutation $\sigma$ of $\{1, \cdots, c\}$ induces an automorphism, also denoted by $\sigma$, on $V$ which is defined by

$$
y_{i 1} \sigma=y_{i \sigma, 1} \text { and } \beta_{i} \sigma=\beta_{i \sigma} .
$$

Again $M \sigma=M$ and so induces an automorphism on $V / M$.

Clearly the characteristic closure of

$$
\left[y_{i_{1} n_{1}}, \cdots, y_{i_{c} n_{c}}\right]^{q^{\prime}} M
$$

where $\left\{i_{1}, \cdots, i_{c}\right\}$ is a permutation of $\{1, \cdots, c\}$ and $q^{\prime}=q / p$, is $S / M$; so it suffices to find such an element in the characteristic closure of $z M$ for every $z$ in $S \backslash M$. The element $z$ can be taken as a product of left normed commutators

$$
\left[y_{1 n_{1}}, y_{i_{2} n_{2}}, \cdots, y_{i_{c} n_{c}}\right]^{q^{\prime}}
$$

where $\left\{i_{2}, \cdots, i_{c}\right\}$ is a permutation of $\{2, \cdots, c\}$. It suffices to show that the closure of such a product in $V$ under the $\gamma_{i}$ and transposing automorphisms (i $j)$ contains some commutator

$$
\left[y_{i_{1} n_{1}}, \cdots, y_{i_{c} n_{c}}\right]^{q^{\prime}}
$$


The product $z$ of commutators can be written as a product of products

$$
u=\prod_{n=1}^{k}\left[\cdots, y_{c n}, \cdots\right]^{d(n) \cdot q^{\prime}} \text {, }
$$

with $0 \leqslant d(n)<p$. Such an element $u$ can be thought of as corresponding to the same element of the field $K$ as the element

$$
\prod_{n=1}^{k} y_{c n}^{d(n)} \Phi
$$

of $V_{c} / \Phi$ does. Then, each of the elements

$$
\prod_{j=0}^{k-1}\left(\beta_{c}^{-f} u \beta_{c}^{f}\right) \gamma_{c}^{j}
$$

for $f \in\{0, \cdots, k-1\}$ can be thought of as corresponding to an element of the fixed field of the automorphism $x \mapsto x^{p}$ of $K$, that is to an element of the prime field of $K$. A Vandermonde argument shows that at least one of these elements, (2.6), is nonzero, if one of $d(1), \cdots, d(n)$ is nonzero. Thus it suffices to consider nontrivial products of commutators in which $y_{c 1}$ is the only entry with first subscript $c$. Repetition of this argument with the first subscripts $c-1, c-2, \cdots, 1$ in turn yields that it suffices to consider nontrivial products of the form

$$
v=\prod_{i=2}^{c}\left[y_{11}, y_{i 1}, y_{21}, \cdots, y_{(i-1), 1}, y_{(i+1), 1}, \cdots, y_{c 1}\right]^{e(i) \cdot q^{\prime}},
$$

where $0 \leqslant e(i)<p$. If $e(i) \neq e(j)$, then $v(i j) v^{-1}$ is nontrivial and is a power of a commutator of the form of (2.3); while if all the $e(i)$ are equal, then $v(12) v^{-1}$ is nontrivial and is a power of a commutator of the form of (2.3). This completes the proof of part (iii) of Theorem II.

The analysis above in Theorem II leads to a proof of Theorem I in the following manner. By part (iii) of Theorem II every critical group $C$ in a proper subvariety of $\mathcal{M} \boldsymbol{r}_{c, q} \mathfrak{Q}_{m}$ satisfies either that Fit $C$ does not generate $\left.\mathcal{M}\right\}_{c, q}$ or that $C /$ Fit $C$ is not a $c$-fold direct power of a cyclic group of order $m$. By part (i) of Theorem II such a critical group $C$ must satisfy either part (a) or part (b) in (ii) of Theorm II. Thus any critical group from a proper subvariety of $M_{c, q} \mathfrak{A}_{m}$ is contained in the proper subvariety mentioned in (ii) of Theorem II which is the proper subvariety of $\mathcal{M l} q_{c, q} \mathfrak{A}_{m}$ determined by the word $\omega$. Hence any proper subvariety of the variety $\mathcal{M i}_{c, q} \mathfrak{A}_{m}$ has $\omega$ as a law. Therefore it is impossible for the variety $\mathfrak{M H}_{c, q} \mathfrak{A}_{m}$ to be decomposed into the join of proper subvarieties.

3. Acknowledgements. The author wishes to express his gratitude to Professor Paul M. Weichsel for his patient guidance and encouragement throughout this research. 
The author also wishes to thank the referee for his numerous suggestions on the organization of the paper and statements of the results. Also the author wishes to express his thanks to the referee for simplifying many of the author's arguments.

\section{REFERENCES}

1. Warren Brisley, On varieties of metabelian p-groups, and their laws, J. Austral. Math. Soc. 7 (1967), 64-80. MR 34 \#7646.

2. R. G. Burns, Verbal wreath products and certain product varieties of groups, J. Austral. Math. Soc. 7 (1967), 356-374. MR 36 \#249.

3. - Some applications of wreath products of groups, Ph.D. Thesis, Australian National University, Canberra, Australia, 1966.

4. P. J. Cossey, On varieties of A-groups, Ph.D. Thesis, Australian National University, Canberra, Australia, 1966.

5. Hanna Neumann, Varieties of groups, Ergebnisse der Mathematik und ihrer Grenzgebiete, Band 37, Springer-Verlag, New York, 1967. MR 35 \#6734.

6. M. F. Newman, On a class of metabelian groups, Proc. London Math. Soc. (3) 10 (1960), 354-364. MR 22 \#8074.

7. D. R. Taunt, Remarks on the isomorphism problem in theories of construction of finite groups, Proc. Cambridge Philos. Soc. 51 (1955), 16-24. MR 16, 792.

8. Paul M. Weichsel, On critical p-groups, Proc. London Math. Soc. (3) 14 (1964), 83-100. MR 28 \#3092.

9. James J. Woeppel, Finite groups generating a product variety, Ph.D. Thesis, University of Illinois at Urbana-Champaign, Urbana, Illinois, 1970.

DIVISION OF NATURAL SCIENCES, INDIANA UNIVERSITY SOUTHEAST, NEW ALBANY, INDIANA 47150 